\title{
AN OPTIMAL FACE RECOGINITION TOOL
}

\author{
Marri.Lingareddy ${ }^{1}$, D. Haritha ${ }^{2}$ \\ ${ }^{1}$ Research scholar, ${ }^{2}$ Assistant professor, ECM Department, KL University, AP, India, \\ marrilingareddy@gmail.com.haritha_donavalli@kluniversity.in
}

\begin{abstract}
Face recognition is one of the crucial and important methods for the security purposes. The accuracy of the facial recognition system degrades over time. Therefore the FRS system must be up-to-date. Sometimes the system will not be up-to-date because of unlabelled face images, or because of change in the facial expressions or because of any relevant changes in face of person. Therefore in such cases also the FRS must be self-trained to make the system up-to-date. In this paper a semi-supervised version, based on the selftraining method, of the classical PCA-based face recognition algorithm is proposed to exploit unlabelled data for off-line updating of the Eigen space and the templates. Reported results show that the exploitation of unlabelled data by self-training can substantially improve the performances achieved with a small set of labelled training examples.
\end{abstract}

Index Terms: Eigen faces, Face recognition, face classifier, occluded faces

\section{INTRODUCTION}

Face recognition is a method of automatically identifying or verifying a person's video frame or video source or any digital image. Generally this type of method is used for security purposes and the face its self is used as a key to identify the systems password. Therefore a facial recognition system is a computer application to check the identity of the person. One of the ways to do this is by comparing selected facial features from the image and a facial database.

\subsection{Methods}

There are three different type of techniques are employed to detect the face. They are
I. Traditional method
II. 3-dimensional recognition
III. Skin texture analysis

In traditional method the facial recognition is done based on extracting the landmarks or features from an image of his/her face. Therefore an algorithm is written in such a way that based on the relative position, size, and/or shape of the eyes, nose, cheekbones and jaw are used to search for other images with matching features. Recognition algorithms can be divided into two main approaches, geometric, which look at distinguishing features, or photometric, which is a statistical approach that distils an image into values and compares the values with templates to eliminate variances.

The 3-D face recognition method is a newly emerging method and this method uses 3D sensors to capture information about the shape of the face. This method is used to acquire improved accurate results. Added advantage to this method is that the face recognition is not affected by changes in lighting and can also identify a face from a range of viewing angles, including a profile view.

The skin texture method uses the unique lines, patterns and spots apparent in a person's skin and turns them into a mathematical space. Facial recognition systems are also beginning to be incorporated into unlocking mobile devices. The android market is working with facial recognition and integrating it into their cell phones. They have created an application called Visidon Applock. This application allows you to put a facial recognition lock on any of your applications. This allows you to increase the safety of your private apps and content. Facial recognition technology is already implemented in the iPhoto application for Macintosh.

\subsection{Face Accuracy}

The accuracy of Face Recognition Systems (FRSs) on a user will decrease with time [4][5][6][7]. The decrease in the accuracy is the result of changes in facial appearance because of factors such as ageing, hair-growth, surgery, weight changes, sun-exposure, ancestry, sex, health, disease, drug use, diet, sleep deprivation, and bio-mechanical factors [1][8][9][10]. The appearance changes in user are illustrated with the figure1.

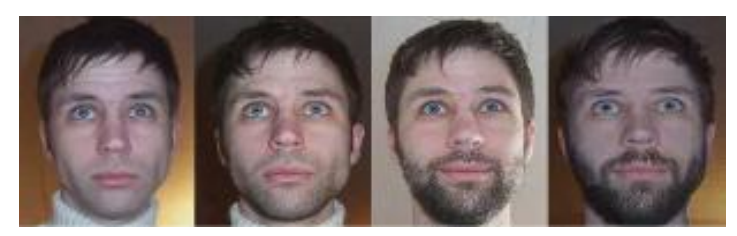

Figure1: Facial appearance in a person due of hair growth 
Therefore to maintain the accuracy of the facial recognition system over time the FRS should be up-to-date. FRS will be up-to-date through the self-update procedure. In this procedure the system is re-tined with the unlabelled data, which is acquired every time a user interacts with the system. The unlabelled facial images are added to the training set according to the predicted label.

\subsection{Algorithm Types}

The method of classifier is used for recognition affects the long term accuracy of the self-updating FRS's. Therefore three different types of algorithms are designed [1]. They are

\author{
i. $\quad$ Eigen faces(EF) \\ ii. Fisher faces(FF) \\ iii. Similarity based fisher faces(SFF)
}

These algorithms are chosen because of their inductive reasoning in their training process. The EF is purely a transductive reasoning and doesn't use inductive reasoning. The FF is a little inductive in nature because it uses a global statistics of the user data to find the best discriminating subspace. SFF is more inductive as user-specific models are built in such a way that they are later used for recognition. Induction is a method which uses training data to find approximations of functions that describe the data.

\section{RELATED WORK}

Considerable research in social psychology has shown that facial expressions help coordinate conversation and have considerably more effect on whether a listener feels liked or disliked than the speaker's spoken words.

Mehrabian indicated that the verbal part of a message contributes only for 7 percent to the effect of the message as a whole. The vocal part contributes for 38 percent, while facial expression of the speaker contributes for 55 percent to the effect of the spoken message. Therefore face recognition is an important addition to computer vision research [1]. Computer vision aims to duplicate the effect of human vision by electronically perceiving and understanding an image.

Computer vision techniques use the results of mathematics, pattern recognition, artificial intelligence, psycho-physiology, computer science, electronics, and other scientific disciplines. Recent advances in image analysis and pattern recognition open up the possibility of automatic detection and classification of emotional and conversational facial signals. Automating facial expression analysis could bring facial expressions into man-machine interaction as a new modality and make interaction tighter and more efficient. Such a system could also make classification of facial expressions widely accessible as a tool for research in behavioral science and medicine [1].

\section{REPRESENTATION OF FACIAL ECOGNITION SYSTEM}

The outline of a typical face recognition system is given figure 2 . This outline carries the characteristics of a typical pattern recognition system.

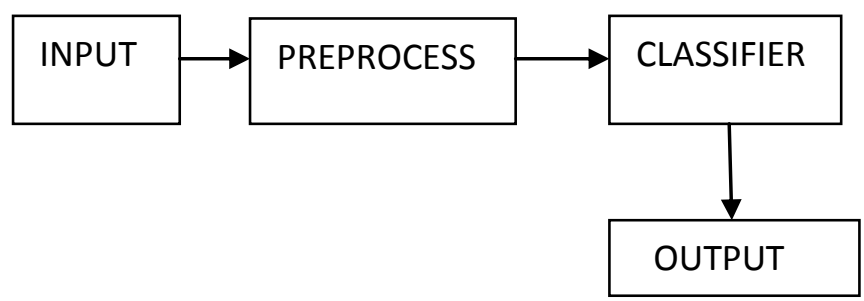

Figure2: Generic representation of facial recognition

There are 4 main blocks that represent the face recognition. In the input block an image is passed to the system for classification. The images vary in format, size and resolution. The image is pre-processed to remove unwanted noise from light and the environment. The image also then is compressed and normalized. The classifier decides whether the image belongs to the face or the non-face class based on the information learned during training. The output image indicates whether the input image is thought to certain or not. Some systems indicate its location.

\section{PATTERN RECOGNITION}

Pattern recognition is a modern day machine intelligence problem with numerous applications in a wide field, including face recognition, character recognition, speech recognition as well as other types of object recognition. Its ability to read text and shapes plays an important role in medical diagnostic science, machine conditioning and monitoring and sorting. The speed of processing is important for its real-time application in banks, stores and voice recognition banks, stores and voice recognition. A basic pattern classification system consists of sensor inputs, feature extraction, selection and classification. Sensors could be microphone, digital camera orographic, features are extracted with statistical, geometrical or descriptor means. The field of pattern recognition is still very much in its infancy, although in recent years some of the barriers that hampered such automated pattern recognition systems have been lifted due to advances in computer hardware providing machines capable of faster and more complex computation. Pattern recognition can be classified as the categorization of input data into identifiable classes via the extraction of significant features or attributes of the data from a background of irrelevant detail. The functional block diagram of an adaptive pattern recognition system is shown in Figure3. 


\section{FACE RECOGNITION}

Face recognition is a pattern recognition task performed specifically on faces. It can be described as classifying a face either "known" or "unknown", after comparing it with stored known individuals. It is desirable to have a system that has the ability of learning to recognize unknown faces.

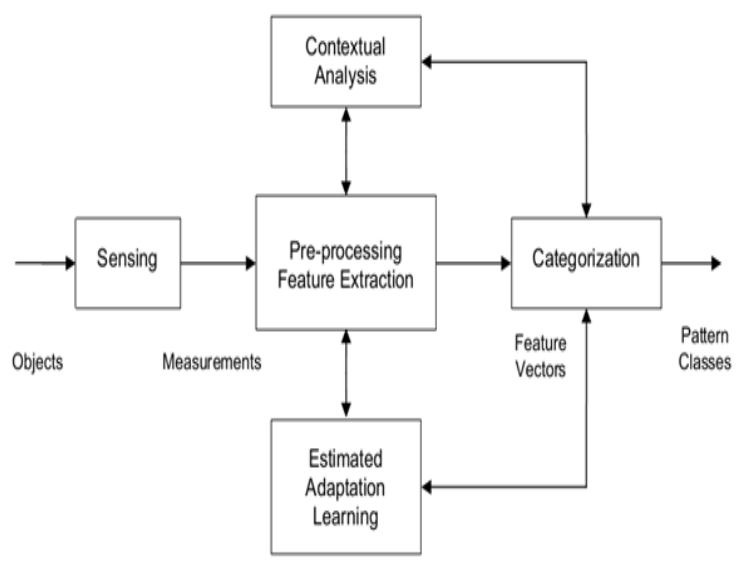

Figure3: Block diagram of Pattern recognition

Face recognition involves comparing an image with a database of stored faces in order to identify the individual in the input image. The related task of face detection has direct relevance to recognition because images must be analyzed and faces identified, before they can be recognized.

\subsection{Types of image conditions}

Face recognition, although a trivial task for the human brain has proved to be extremely difficult to imitate artificially, because although commonalities exist between faces, they can vary considerably in terms of age, skin color, orientation, facial expression and presence official furniture such as glasses or facial hair. The problem is further complicated by differing light conditions, image qualities and geometries, as well as the possibility of partial occlusion and disguise. Hence, a robust face recognition system should be capable of classifying a face image as "known" under the following conditions:

- Scale variance

- Orientation variance

- Illumination variance

- Background variance

- Emotional expression and detail variance

- Noise variance

For basic face recognition systems, some of these effects can be avoided by assuming and ensuring a uniform background and uniform light conditions.

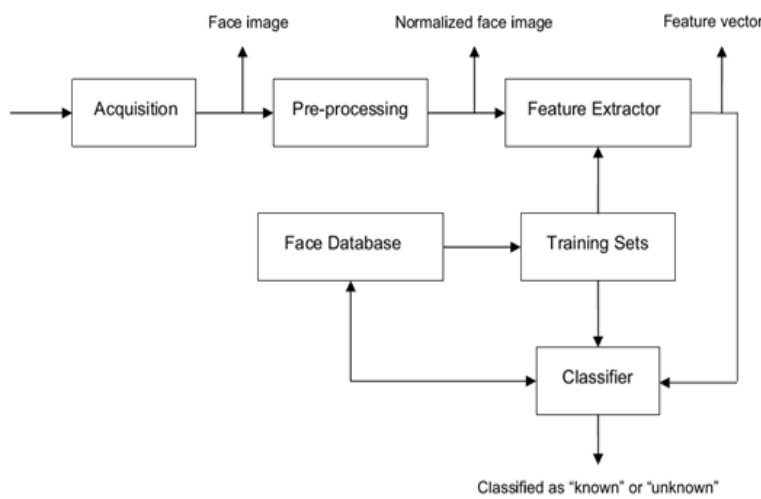

Figure 4: Block diagram of face recognition system

This assumption is acceptable for some applications such as automated separation of nuts from screws on a production line, where light conditions can be controlled and the image background will be uniform. Figure 4 outlines the block diagram of a typical face recognition system.

Face recognition is the most natural, non-intrusive, and userfriendly biometric measure, because it requires no disturbance to the people being identified. Most of the existing systems can successfully recognize faces only when they appear under constrained conditions. The performance will degrade significantly when face images are obtained under variable illumination conditions, facial expressions, poses or perspectives, especially when occlusion happens. Occlusions may be caused by sunglasses, a thick beard, the wearing of a scarf, or expression variations. In this paper, we will focus on how to improve the performance for recognizing occluded faces. In a nutshell, we first complete or repair the occluded faces with our proposed spectral algorithm, and then perform face recognition on the completed images. A face-completion algorithm involves three main procedures: occluded face clustering via the sparse representation, image-based data mining, and face image repairing using the spectral graph theory. Our algorithm is developed on the basis that global and local similarities among different faces are widespread. A damaged face can be considered as a signal sequence, in which each un-occluded pixel is seen as the element. The sequence is matched with faces in our large database via sparse representation, and then the most similar local part to the occluded part is selected after the sparse representationbased clustering. Some other classification algorithms, e.g. the Euclidean distance and Sum-of-Squared-Differences (SSD) based classification, are alternative to this step. However, compared to sparse representation, all the other methods require solving the classification problem via an exhausting search, which is very computationally intensive. The sparse representation method is able to avoid searching all the items in a more efficient way via dynamic programming. 
Finally, in order to provide fusion and natural repairing results, a mathematical model that follows an actual painters' painting procedure, namely guided label learning, is developed using the spectral graph theory. Image completion is one of the most talked-about topics in the Graphics area]. A number of useful tools, e.g. image in painting .Poisson equations, and graph-cuts, have been established for real-scene image completion. But these ideas require too much information about the damaged images, and they are always performed on high-resolution color images. When referring to face recognition, the algorithms are usually developed based on low-resolution gray-scale images, and the traditional algorithms may lose their effectiveness. Hence, as per the Poisson equation, we extend it to the graph-based domain. Due to the flexibility of the graph topology, low-resolution gray-scale images can be coped with quite well.

\subsection{Proposed scheme}

The major contributions of this paper are as follows:

i. We propose a new face reconstruction algorithm based on sparse representation and a graphical model. Our algorithm is established from a new perspective, different from the existing face reconstruction algorithms.

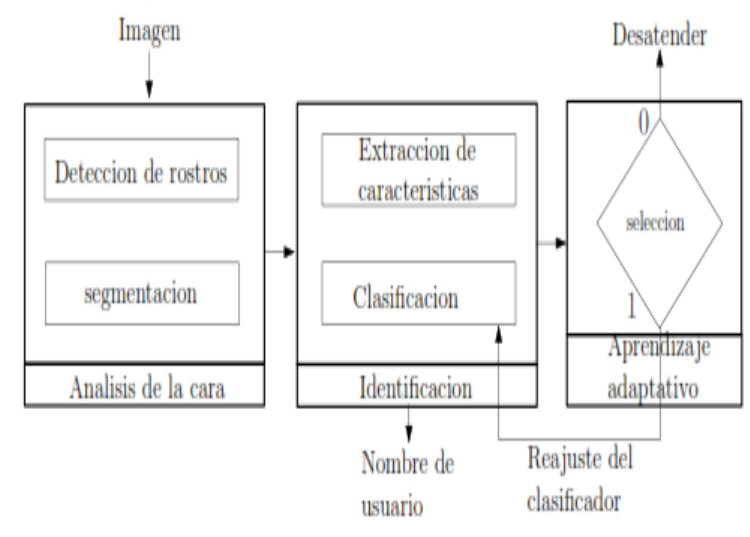

Figure 5: Flow chart of FRS system

ii. The proposed algorithm is able to improve the recognition results dramatically, with an increase of up to $30 \%$ in recognition rate.

Face analysis block: The face analysis block is responsible for detection and segmentation of human faces.

Face detection: To detect faces accurately in real-time, our system employs Viola and Jones face detection procedure [11]. The detector works at a speed of 15 frames per second at 12 resolution levels, while achieving low false acceptance rates.
Face segmentation: For the segmentation of the prominent facial features our system employs Active Shape Models with Invariant Optimal Features (IOFASM) [12]. This algorithm can automatically locate the outlines of eyes, mouth, nose, brows and silhouette contour of the face with an average error of approximately $2 \%$ of the inter-eye distance.

Identification block: The identification block extracts texture from a segmented face and uses it to identify a user.

Facial feature extraction: To make facial texture independent of the facial expression, each image was warped using a piece-wise affine mapping between the result of the segmentation step and the mean shape. The texture parameters for each image were extracted by constructing an image intensity model of an Active Appearance Model [13].

Classification Once the texture parameters are obtained, they are whitened [14] and then a distance-weighted k-Nearest Neighbor classifier (k-NN) is used to identify the user in the image. The distance between two texture vectors is defined as the angle between them. The angle based distance metric was shown to be a good choice for PCA-based algorithms [15]. All the experiments in this paper were performed using the five nearest neighbors $(\mathrm{k}=5)$.

Automatic learning block Every time a facial image has been identified, it goes through an automatic selection process that determines whether it is suitable to be added to the training set of the appropriate user. The selection process deems two categories of images to be unsuitable for automatic learning:

1) images with outdated facial appearance,

2) Images where the algorithms used in our system produce unreliable results.

\section{ANALYSIS OF THE ALGORITHM USED}

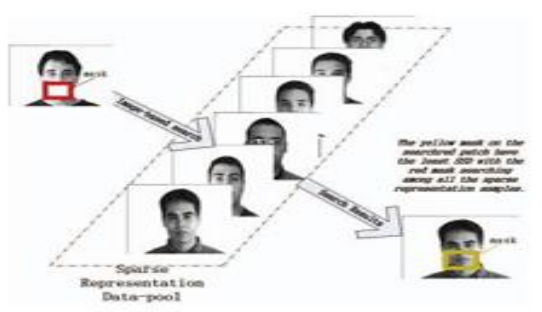

Figure6: Damaged face representation using graph cut

In this algorithm, we first cluster the images which have a similar sparse representation to the query image. Then, these clustered images are used to find the best-matched patch for guiding the synthesis of the missing parts. The patch obtained is used to guide the in-painting work of the damaged part of the occluded face. Hence, the searched patch should have a similar local property as the damaged part. The target region, which needs to be filled in the damaged face image $F$, is de- 
noted as R. The goal is to find the best-matched patch in the sparse representation data-pool, which fits its neighborhood $\mathrm{R}$ with the least SSD. This procedure is shown in Figure 6.

The region of missing pixels is defined as $\mathrm{R}$, and the mask region as $\mathbf{J}$, on a damaged face image. Mathematically, mask $\mathbf{J}$ is an expanded region of the damaged patch. We expand the boundary of $\mathrm{R}$ by $\mathrm{h}$ pixels and define it as:

$$
J(i, j)=0, \text { if }(d(i, j)>h) \operatorname{or}((i, j) \in R), 1, I d(i, j)<h .(1)
$$

The mask $\mathbf{J}$ is painted as a thin band surrounding the missing region R. $d(i, j)$ indicates the distance between the pixel $(i, j)$ and its nearest pixel in the damaged/occluded region $\mathrm{R}$.

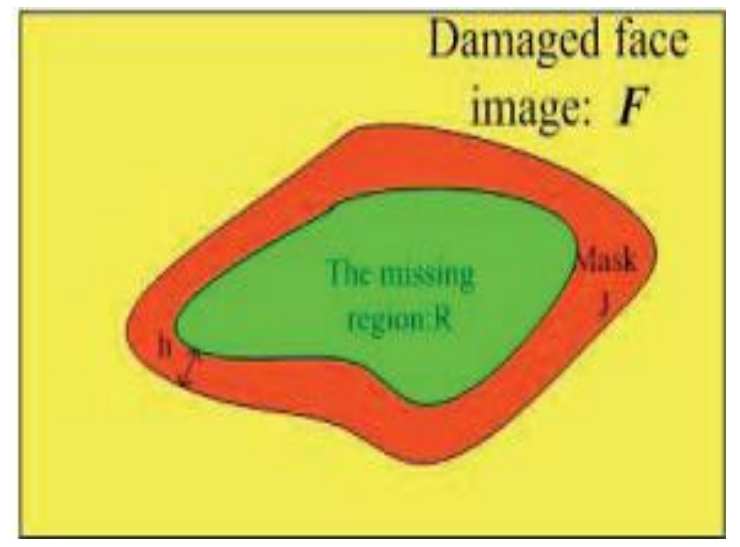

Figure7: The missing region $\mathrm{R}$ and masked region $\mathrm{J}$ of damaged face $\mathrm{F}$

\section{PARTIALLY OCCLUDED FACE OMPLETION AND RECOGNITION}

In this section, we will apply the completion algorithm on partially occluded face images. The occluded part of the face is detected using the graph cut, which is a common method for image segmentation. Consequently, the proposed spectral graphic based image completion algorithm is employed for each damaged faces.

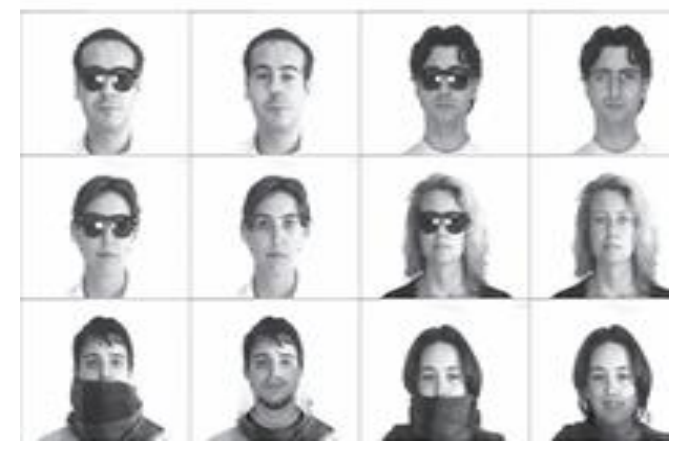

Figure9: AR database for occluded faces
Some repaired results are shown as the second and the fourth column in Figure9. The repairing algorithm is able to provide a fusion and produce natural results. The recognition experiment was also developed based on the AR database. For training, 847 un-occluded images (121 subjects, 7 images per subject) were used. For testing, the occluded faces were divided into two subsets, denoted as AR1 and AR2. These two sets included images with sun-glasses and images with a scarf, respectively. The two methods common face recognition algorithms, the PCA and Gabor methods, with and without using the algorithm to evaluate the recognition performance.

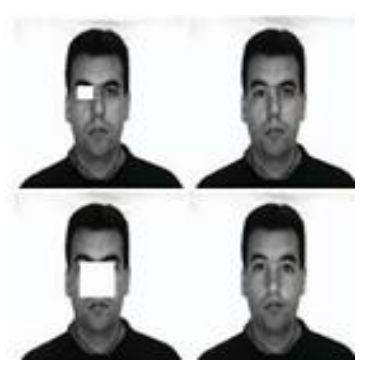

Figure10: Synthetic partially occluded faces

For testing, synthetic occlusions were added to 484 images. As shown in Figure9, the location of the occlusion is randomly selected. And the curves of the recognition rates with different amounts of occlusion are shown in Fig. 6. The occlusion percentages range from $0 \%$ to $50 \%$, in $12.5 \%$ additions.

\subsection{Training and updating procedure}

The k-NN classifier was trained with the texture parameters obtained from the 472 images in the Training set and their corresponding user IDs.

Using the images in the Update set (in chronological order), three types of updates were performed to the Training set [2]:

i. UC. In this case, updates were performed with the total confidence, $\mathrm{C}$.

ii. $\quad \mathrm{UC}_{\mathrm{c}}$. The update process is similar to that performed in [3]. In this scenario, if Cc computed for an image in the Update set is greater than $\mathrm{C}$ of any of the images in the Training set, then the image will be added to the Training set. Otherwise, the image is ignored.

iii. $\mathrm{UC}_{\mathrm{t}}$. This is a trivial update strategy where newer images from Update set replace older ones in the Training set. In other words, if an image has a $\mathrm{Ct}$ value greater than that of any of the images in the Training set, then it is added to the Training set.

\section{CONCLUSIONS}

Face recognition is both a challenging and important recognition technique among all the biometric techniques; face recognition approach possesses one great advantage, which is its user-friendliness. 
As face recognition system has become an important method for security purpose the FRS system was made up-to-date using self-trained method.

In this paper the classical PCA-based face recognition algorithm \& Guided labelled algorithm are used so that the face is recognized in any of the cases (Such as age, growth of hair or beard, any of the blurred images, or the low lighting images and finally different pose of the faces).

Self-training performances are achieved with a small set of labelled training examples. So the self-trained is used to keep the system up-to-date and made the better FRS.

\section{RESULTS}

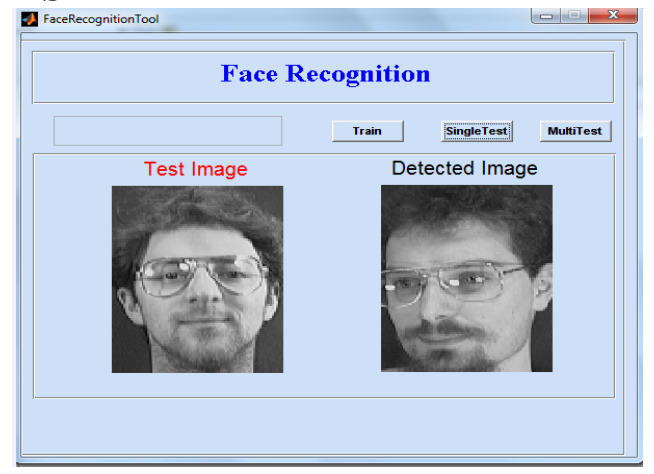

Figure 11: Face recognition is done even in growth of beard

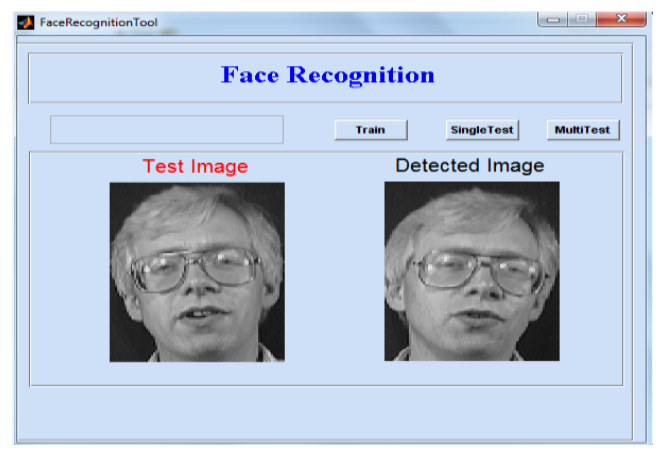

Figure 12: Detected image when the age is grown

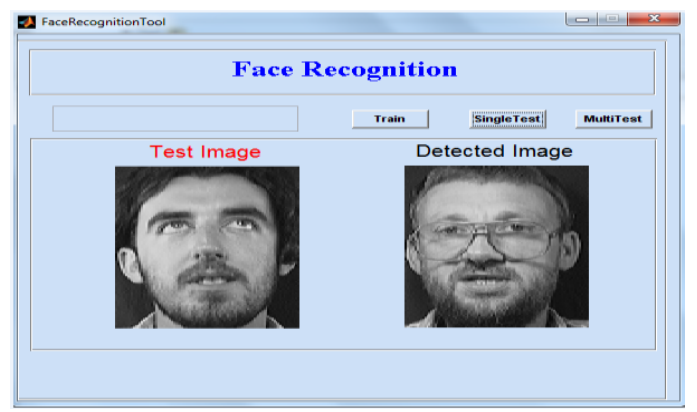

Figure 13: Face recognition is done when the person is aged $\&$ having spectacles on his face

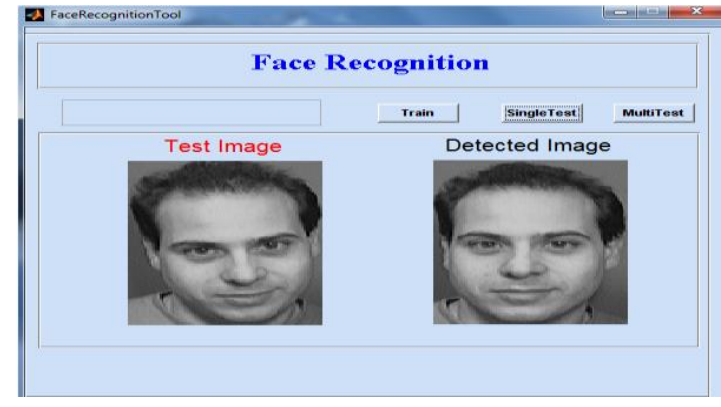

Figure 14: Face recognition is done even for blurred images

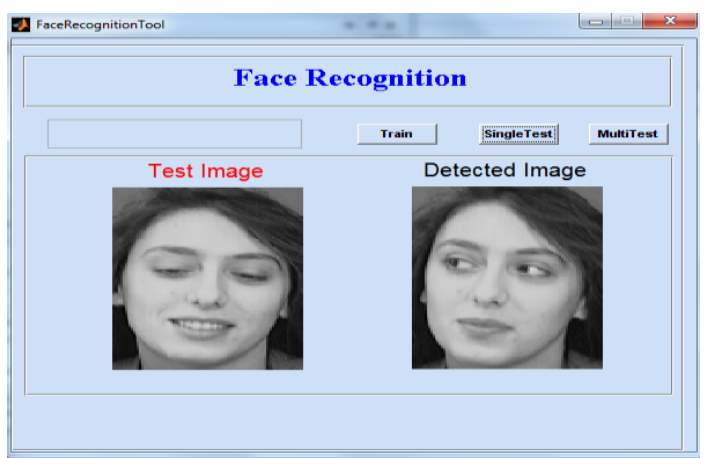

Figure 15: Face recognition is also done when the pose is changed

\section{REFERENCES}

[1] An Experimental Evaluation of Three Classifiers for Use in Self-Updating Face ecognition Systems SriKaushik Pavani, Federico M. Sukno, David DelgadoGomez, Constantine Butakoff, Xavier Planes, and Alejandro F. Frangi

[2] A Confidence-based Update Rule for Self-Updating Human Face Recognition Systems Sri-Kaushik Pavani 1;2, Federico M.

Sukno 2;1, Constantine Butako ${ }^{\circledR}$, Xavier Planes 1;2 and Alejandro F. Frangi

[3] D. Mou, R. Schweer, and A. Rothermel. Automatic databases for unsupervised face recognition.In CVPRW 2004, pages $90\{97\}$.

[4] P. J. Phillips, P. Grother, R. Micheals, D. M. Blackburn, E. Tabassi, and M. one, "Face Recognit. Vendor Test 2002: Evaluation Report," National Inst. Standards Technol., Tech. Rep. NISTIR 6965, 2003.

[5] P. J. Flynn, K. W. Bowyer, and P. J. Phillips, "Assessment of time dependency in face recognition: An initial study," in Proc. 4th Int. Conf. Audio- and Video-Based Biometric Person Authentication. Lecture Notes in Computer. Science, Guildford, U.K., 2003, vol. 2688, pp. 44-51.

[6] H. Wang and P. J. Flynn, "Experimental evaluation of eye location ac- curacies and time-lapse effects on face 
recognition systems," in Proc. 5th Int. Conf. Audio- and Video-Based Biometric Person Authentica- tion. Lecture Notes in Comput. Science, New York, NY, 2005, vol. 3546, pp. 627-636.

[7] H.Ling,S.Soatto,N.Ramanathan,andD.W.Jacobs, “Astud yofface recognition as people age," in Proc. 11th IEEE Int. Conf. Comput. Vis., Rio de Janeiro, Brazil, 2007, pp. $1-8$.

[8] N. Ramanathan, R. Chellappa, and S. Biswas, "Computational methods for modeling facial aging: A survey," J. Vis. Lang. omput., vol. 20, no. 3, pp. 131144, 2009, 1.

[9] L. Millsopp, L. Brandom, G. Humphris, D. Lowe, C. Stat, and S. Rogers, "Facial appearance after operations for oral and oropharyngeal cancer: A comparison of casenotes and patient-completed questionnaire," Brit. J. Oral Maxillofacial Surg., vol. 44, no. 5, pp. 358363,2006.

[10] M. A. Taister, S. D. Holliday, and H. I. M. Borrman, "Comments on facial aging in law enforcement investigation," Forensic Sci. Commun., vol. 2, no. 2, 2000 [Online]. Available: http://www.fbi.gov/about-us/ lab/forensic-science-

communications/fsc/april2000/taister.htm

[11] P. Viola and M. Jones. Robust real-time face detection. Int. J. Comput. Vision, 57(2):137\{154, 2004\}.

[12] F. Sukno et al. Active shape models with invariant optimal features. IEEE Trans. Pattern Anal. Mach. Intell., 29(7):1105\{1117, 2007\}.

[13] T. Cootes, G. Edwards, and C. Taylor. Active appearance models. IEEE Trans. Pattern Anal. Mach. Intell., 23(6):681\{685, 2001\}.

[14] P. Navarrete and J. Ruiz del Solar. Comparative study between di®erent

eigenspace-based approaches for face recognition. In FS 2002, pages $178\{184\}$.

[15] V. Perlibakas. Distance measures for PCA-based face recognition. Pattern Recogn.Lett., 25(6):711\{724, $2004\}$.

\section{BIOGRAPHIES:}

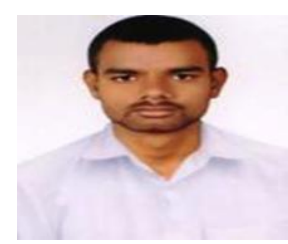

M. Linga Reddy has received his B.Tech degree in ECE from ACE in 2011. He is presently pursuing M.Tech in Embedded systems in E.C.M. Department from KL University since July 2011.

D. Haritha, Assistant professor, ECM Department, KL University AP, India, haritha_donavalli@kluniversity.in 